\title{
Non-Real Time Ultrasound-Guided Spinal/Epidural Anesthesia for C-Section in an Obstetric Patient with Instrumented Lumbar Spine-A Single-Operator Reliable Method: A Case Report
}

\section{Ramirez-Paesano Carlos $^{1 *}$ and Hachoue-Saliba Zeina ${ }^{2}$}

${ }^{1}$ Service of Anesthesiology (Anestalia), Centro Médico Teknon, Calle Vilana 12, Barcelona, Spain

${ }^{2}$ Rehabilitation and Physical Medicine Resident, Hospital Universitario La Paz, Paseo La Castellana, Spain

\begin{abstract}
Background: The benefits of epidural/spinal anesthesia during C-section delivery are well known. Instrumented spines have been a challenge for performing these techniques. We describe the case of an obstetric patient with previous spine surgery programmed for $\mathrm{C}$-section. Three years ago, for her first delivery the patient underwent $\mathrm{C}$-section managed by general anesthesia due to impossibility to perform neuroaxial block. Because of obtaining low newborn score and remarkable pain during the post-operatory period, she requested conductive anesthesia for her second baby delivery.
\end{abstract}

Case presentation: Pre-puncture ultrasound evaluation was made to explore spinal structures, and lumbar sonoanatomy was correlated with previous X-ray images of lumbar surgery to decide the best levels for punctures. Then, the echo-predetermined points of entry for the needles were marked on the patient's back using a skin marker to perform the anesthetic procedure by usual puncture techniques. A non-real time ultrasound-guided Combined Spinal/Epidural Anesthesia (CSE) was successfully achieved with total mother satisfaction and excellent neonatal outcome.

Discussion and conclusion: The knowledge of spinal sonoanatomy should be a part of all anesthesiologists' training. It facilitates enormously neuroaxial anesthetic techniques in difficult cases increasing the rate of success and lowering the number of attempts. The non-real time US-guided method does not demand extraordinary skills from anesthesiologists neither necessity of US-probe/needle coordination providing a reliable and non-cumbersome option for a single operator.

Keywords: Instrumented spine; Obstetric anesthesia; Ultrasound; Single-operator; Non-real time ultrasound guidance; Neuroaxial anesthesia; CSE

Abbreviations: US: Ultrasound; CSE: Combined Spinal/Epidural Anesthesia; SLFD: Skin-Ligamentum Flavum/Dura Complex Distance; PDPH: Post-Dural Puncture Headache; G: Gauge, VAS: Visual Analog Scale; MRI: Magnetic Resonance Image; LA: Local Anesthetic

\section{Introduction}

Neuroaxial anesthesia is currently considered the gold standard anesthetic technique in obstetric patients because it has been associated with lower maternal morbi-mortality since it has been routinely applied for many decades. Compared to general anesthesia, the usage of these techniques (spinal, epidural or CSE) has reduced bronco-aspiration and failed intubation events (both considered fatal anesthesia related complications) [1] as well as provided better neonatal outcome.

Previous spinal surgery has classically been considered an obstacle for applying conductive anesthesia due to difficulties in performing neuroaxial techniques by blind approach in these patients, so most anesthesiologists prefer general anesthesia despite its well-known risks in obstetrics patients.

Now-a-days, spinal surgical antecedents may stop being a significant obstacle for spinal or/and epidural anesthesia thanks to the advances in the practice of spinal sonoanatomy. We present a case report of an obstetric patient with previous spine surgery undergoing cesarean delivery who successfully received non-real time ultrasound-guided CSE anesthesia.

\section{Case Presentation}

A 37 -year-old female patient who weighs $68 \mathrm{~kg}$, a 39 weeks/4 days pregnancy not in labor, programmed for elective cesarean delivery (III pregnancies, I abortion, I Cesarean). Six years ago, the patient suffered from back pain due to spondylolisthesis (L4-S1) with lumbar instability, so she underwent spinal surgery with pedicle screw fixation. Three years ago, the patient underwent $\mathrm{C}$-section delivery and received general anesthesia with remarkable pain in the post-operatory period and no contact with her newborn for 2 days because he was sent to the neonatal care unit owing to low APGAR score. Due to her discomfort and stressful experiences, the patient "begged" us to avoid general anesthesia technique and requested better pain management; she was also afraid of the neonatal outcome. The risks of both anesthetics techniques and the probability of general anesthesia in case of CSE anesthesia failure, accidental dural-puncture, and Post-dural puncture headache (PDPH) were explained to the patient and she signed the consent informed document. We ask for all radiological documents related with her spinal surgery in order to be able to do an X-Ray/Echo correlation of findings.

On the physical exam, a scar from L3 to the sacrum was noted. Additionally, the lumbar spinous processes could not be palpated, so it was impossible to determine the intervertebral spaces or the neuroaxial midline by palpation. X-rays evaluation showed transpedicular instrumentation with bilateral bars extended from L4 to S1 (Figure 1).

The ultrasound (US) scanning was performed with a M-Turbo ultrasound machine (SonoSite) with a 2 to $5.5-\mathrm{MHz}$ broadband multifrequency probe. For the longitudinal approach, the probe was

*Corresponding author: Ramirez-Paesano C, Service of Anesthesiology (Anestalia), Centro Médico Teknon, Calle Vilana 12, Barcelona, Spain, Tel: 0034-684-37-6060 E-mail: cramirez@anestalia.com

Received November 07, 2018; Accepted November 12, 2018; Published November 14, 2018

Citation: Ramirez-Paesano C, Hachoue-Saliba Z (2018) Non-Real Time Ultrasound-Guided Spinal/Epidural Anesthesia for C-Section in an Obstetric Patien with Instrumented Lumbar Spine-A Single-Operator Reliable Method: A Case Report. J Clin Case Rep 8: 1189. doi: 10.4172/2165-7920.10001189

Copyright: () 2018 Ramirez-Paesano C, et al. This is an open-access article distributed under the terms of the Creative Commons Attribution License, which permits unrestricted use, distribution, and reproduction in any medium, provided the original author and source are credited. 
Citation: Ramirez-Paesano C, Hachoue-Saliba Z (2018) Non-Real Time Ultrasound-Guided Spinal/Epidural Anesthesia for C-Section in an Obstetric Patient with Instrumented Lumbar Spine-A Single-Operator Reliable Method: A Case Report. J Clin Case Rep 8: 1189. doi: 10.4172/21657920.10001189

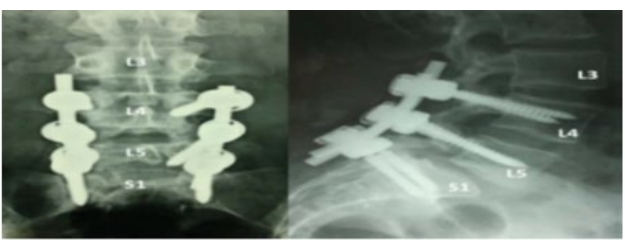

Figure 1: Previous lumbar X-rays: Instrumentation spine from L4 to S1.

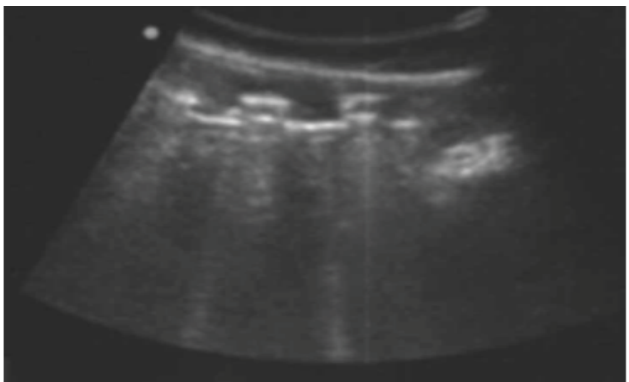

Figure 2: US exploration. Longitudinal axis (Parasagittal view): Chirurgical instrumentation artifacts can be noticed.

placed parasagittally, angled toward the midline. The sacrum was identified, and then the probe was moved cephalad to identify the vertebral interspaces. During pre-puncture ultrasonic evaluation of neuroaxis, we observed with difficulty the spinous processes images. The level of L1-L2, L2-L3, L3-4 and L4-5 intervertebral spaces were identified via US by transverse and longitudinal approach.

The predetermined point of entry for the introducer needle was marked on the patient's back using a skin marker. A transverse line was drawn with a disposable skin marker at either the L1-L2, L2L3, L3-4 and L4-5 interspace. The probe then was rotated $90^{\circ}$ and placed transversely to determine the midline, which was marked by a longitudinal line. The point of needle insertion was determined as the intersection of the longitudinal and transverse lines. The probe was tilted slightly up and down to ensure an enough echogenic window at the selected intervertebral level.

Instrumentation artifacts at the lateral articular processes corresponding to the surgical area were well identified. L4-L5 space was considered to have the best ultrasonic view of spinal cannal despite partial disruption of the flavum ligament (Figures 2 and 3). We measured the skin-ligamentum flavum/dura complex distance (SLFD). The sonoanatomy was normal at L1-L2 space (Figure 4). So first, we decided to put an epidural catheter for post-operatory PCA analgesia into L1-L2 space, and then to give spinal anesthesia for surgery at level of L4-L5 because it had an appropriate spinal cannal area (Figure 3).

In sitting position, and after antiseptic technique with iodine povidone, infiltrative anesthesia with lidocaine $2 \%$ was used onto skin marked points selected to achieve the spaces L1-L2 and L4-L5. The epidural space (L1-L2) was located by "the loss of resistance" test with 18 Gauge $(\mathrm{G})$ Touhy epidural needle and an $20 \mathrm{G}$ epidural catheter was left for post-operatory pain management (only the test dose was administered via epidural catheter in this moment) (Figures 5A and 5B).

Then, to overcome fibrotic tissue due to lumbar surgery, a $16 \mathrm{G}$ Tuohy epidural needle was used as a guide to facilitate the pencil point needle progression through the space at L4-L5 to achieve a spinal anesthesia. The above mentioned Tuohy needle was inserted until a depth equivalent to SLFD-1 cms. to avoid accidental dura-puncture.

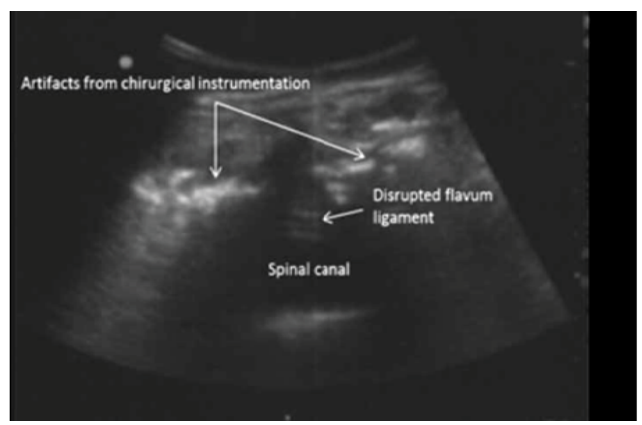

Figure 3: US exploration. Transverse view (short axis): Very bad acoustic window, lack of transverses apophysis identification and disrupted flavum ligament due to previous surgery. This image corresponds to the selected L4-L5 space for spinal anesthesia.

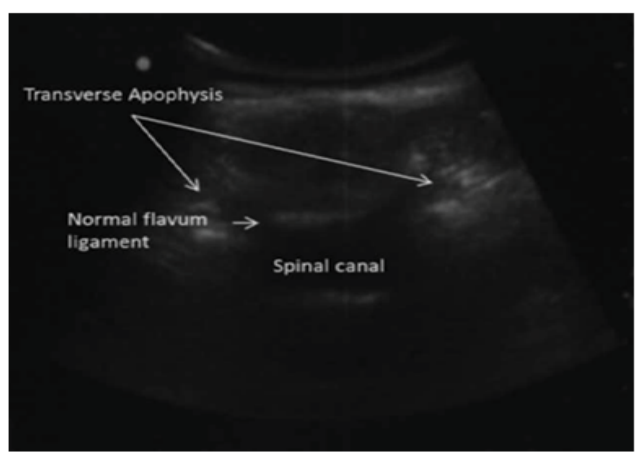

Figure 4: Normal L1-L2 sonoanatomy: Selected space for epidural catheter placement in this case.

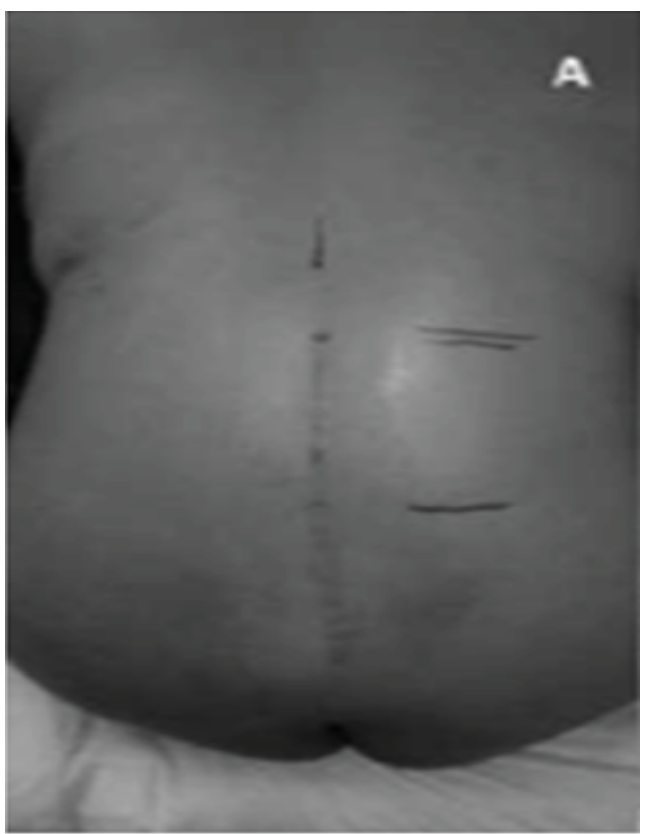

Figure 5A: Marked skin for referring puncture points posterior to prepuncture ultrasound evaluation.

Following, a $26 \mathrm{G}$ pencil point spinal needle was completely threaded through the 16G Touhy needle until obtaining cerebrospinal fluid (CSF); Then, isobaric Bupivacaine 0.5\% $7.5 \mathrm{mg}$ plus Fentanyl $25 \mu \mathrm{gr}$ was 
Citation: Ramirez-Paesano C, Hachoue-Saliba Z (2018) Non-Real Time Ultrasound-Guided Spinal/Epidural Anesthesia for C-Section in an Obstetric Patient with Instrumented Lumbar Spine-A Single-Operator Reliable Method: A Case Report. J Clin Case Rep 8: 1189. doi: 10.4172/21657920.10001189

Page 3 of 4

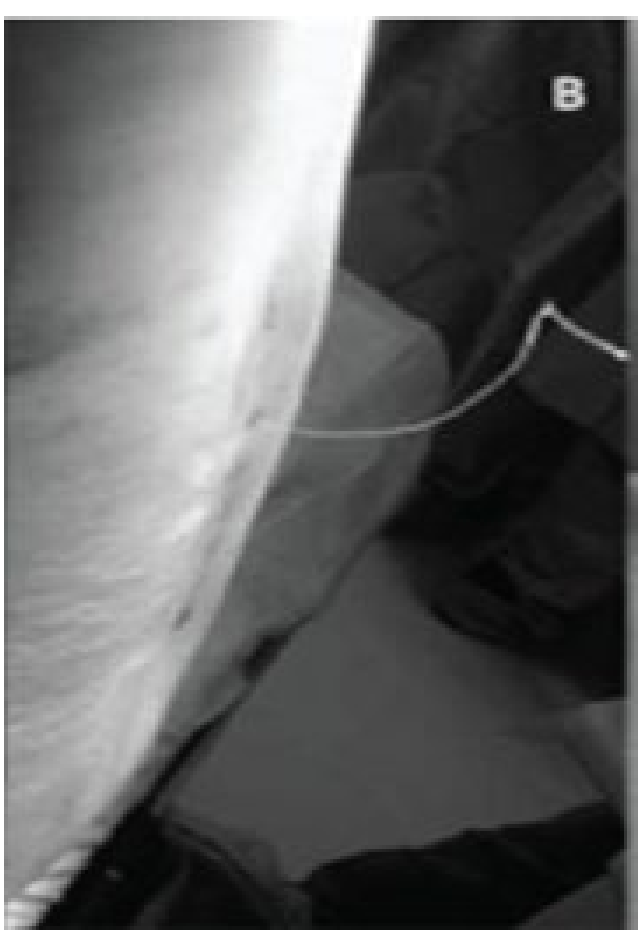

Figure 5B: Epidural catheter for post-cesarean analgesia in L1-L2

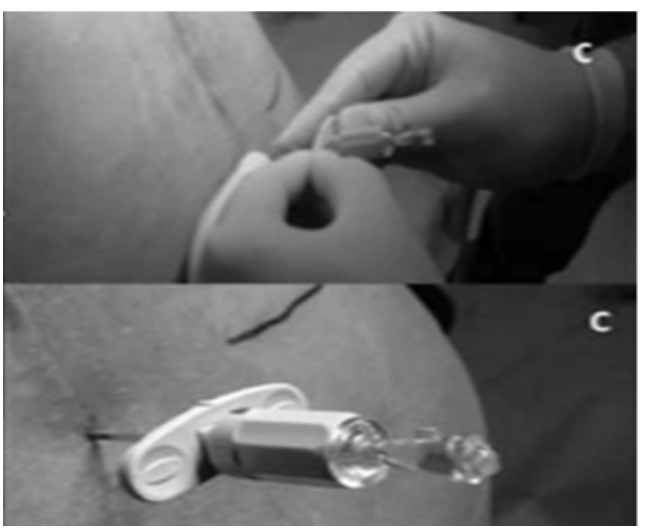

Figure 5C: LCR coming out of Spinal 26 G needle using an $18 \mathrm{G}$ Tuohy epidural needle as guide to overcome fibrosis tissue in L4-L5.

used for anesthesia (Figure 5C). Sensitive blockade was established at T4 before incision (Pfannestiel). Eventual hypotension (under 10\% of basal measure) was corrected with phenylephrine 25-50 $\mu$ gr. Neonatal outcome was optimum with an APGAR score of 8 and 9 points at the first and fifth minutes after delivery.

After surgery, post-operative pain was managed by continuous epidural infusion $(4 \mathrm{ml} / \mathrm{h}$ ) plus PCA ( $4 \mathrm{ml}$ bolus, max. 2 bolus per hour, block-out of 20 minutes) with a mixture of Fentanyl $3 \mu \mathrm{g} / \mathrm{ml}$ and Bupivacaine $0.0625 \%$ during the next $72 \mathrm{hrs}$. Visual Analog Scale (VAS) was under 3/10 during her hospital stay. Our patient was very satisfied with the anesthetic and post-operatory pain management and the overall outcome.

\section{Discussion and Conclusion}

Although, radiological methods such as fluoroscopy, tomography scan or MRI can provide accurate information about the place of puncture and the anatomic characteristics of the spine [2] they are expensive, unpractical and inaccessible in the labor room. On the other hand, ultrasound equipment is always present near or in a labor area and can be easily moved beside the pregnant patient.

Advantages along with the increasing knowledge acquired by anesthesiologists about spine-sonoanatomy, make ultrasound a very attractive tool to facilitate the accessibility of instrumented spines for neuroaxial anesthesia. However, it is mandatory to evaluate previous $\mathrm{X}$-ray images in order to establish correlation with ultrasonic findings. Under these circumstances, it is important to clearly explain to patients the "pros and cons" of trying neuroaxial via and the possibility of failure. It is also mandatory to get the consent informed document signed.

Using ultrasound, it is possible to exactly identify the intervertebral spaces, the ligamentum flavum-dura complex, the medullar/spinal canal and to measure the distance between the skin and the ligamentum flavum-dura complex (SLFD) $[3,4]$. In our patient, we were able to identify the intervertebral spaces by counting the structures from sacrum.

Because of the lumbar scar and lack of spinal bone landmark due to the spine surgery, it was very difficult to identify any space by palpation. Several studies have compared the capability of identification of spinal intervertebral spaces with ultrasound $v$ s. palpation method for spinal/ epidural anesthesia, and higher rate of success have been founded in ultrasound guided groups $[3,5]$.

Fixation materials were easily recognizable by US imaging and the absence of bone structures well correlates with surgical antecedents. The presence of gaps in the ligamentum flavum-dura complex appears to be related with accidental dural-puncture events during epidural anesthesia [6,7]. For this reason, spinal anesthesia would be preferred in cases where an abnormal ligamentum flavum-dura complex is identified. We identified by transverse view (short axis) on US exploration, abnormalities of transverses apophysis and an irregular flavum ligament-dura complex line that was interpreted as a disrupted flavum ligament due to previous spinal surgery. This image corresponded to the selected L4-L5 space for spinal anesthesia (Figure 3). This feature was important to decide for a spinal technique to avoid accidental dural-puncture with epidural technique.

Instrumentation and fibrosis were an issue. Fibrosis produced from lumbar surgical manipulation could cause irregular distribution of local anesthetic (LA) into the epidural space. Therefore, spinal anesthesia was the most suitable technique for this patient although there is always the possibility of failure with this technique [8]. Moreover, fibrosis can make it difficult for very fine spinal pencil-point needles to pass through the tissue. For that reason, we decided to use the $16 \mathrm{G}$ Touhy needle as a guide to avoid problems in getting into spinal canal and obtain CSF. The US exploration has been used in cases were no external landmarks are found for providing spinal anesthesia [9].

An intact sonoanatomy of L1-L2 made it possible to leave an epidural catheter for post-operatory analgesia giving our patient all the well-known benefits of satisfactory post-operatory pain control. We were afraid of providing CSE at L1-L2 due to potential accidental injury of the conus medullaris [2]. Neither we wanted to expose the patient to any possibility of suboptimal epidural anesthesia due to irregular distribution of the LA because of epidural fibrosis. Majeed et al. [10] published the use of spinal catheter for continuous spinal anesthesia technique for cesarean section in a 28 -week primigravida parturient with thoracolumbar scoliosis corrected with Harringtons 
Citation: Ramirez-Paesano C, Hachoue-Saliba Z (2018) Non-Real Time Ultrasound-Guided Spinal/Epidural Anesthesia for C-Section in an Obstetric Patient with Instrumented Lumbar Spine-A Single-Operator Reliable Method: A Case Report. J Clin Case Rep 8: 1189. doi: 10.4172/21657920.10001189

Page 4 of 4

rod surgery. They used a $20 \mathrm{G}$ epidural catheter put into place through an $18 \mathrm{G}$ Touhy needle, maybe to titrate the dose of LA. Apparently, they did not have another option than to establish a continuous anesthesia/analgesia although, with this technique, PDPH is a concern for potential postoperative complication, even more so, because of its difficult management if PDPH becomes severe. Fortunately, we did not have difficulties to put a catheter into an intervertebral level with normal epidural space identified by US.

Tran et al. [11] have described a single-operator real-time USguidance. However, we considered this technique very cumbersome, demanding extraordinary skill from the operator regarding the handling of the probe/needle and time consuming. We performed a non-real time US/Puncture technique described by Arzola et al. [3,4], which makes it possible to mark the "coordinates" superficially on the skin, to preserve optimal antisepsis and performing the puncture at the proper site using the same epidural, spinal or CSE technique as usual. Another concern with single-operator real-time is the theoretical possibility of dragging echogenic-gel with the tip of the needle towards neurological structures or into CSF.

We conclude that knowledge of spinal sonoanatomy should be a part of all anesthesiologists' training. It facilitates neuroaxial anesthetic techniques in difficult, if not impossible, cases increasing the rate of success, lowering the number of attempts, having more anesthetic options and offering our pregnant patients the best anesthesia they could receive.

The non-real time US-guided method does not demand extraordinary skills from anesthesiologists neither necessity of USprobe/needle coordination providing an easy and reliable option for a single operator.

\section{Declarations}

Ethics approval, consent to participate. The ethics committee of the hospital (Clínica El Avila) approved to use the clinical information of the case following strict compliance with the regulations of personal data protection. The authors declare that no patient data appear in this article. The patient signed the informed consent document and gave her permission to publish the figures and photos.

\section{Protection of human and animal subjects}

The authors declare that no experiments were performed on humans or animals for this study.

\section{Consent for publication}

The patient signed the written informed consent document and gave her permission to publish the figures and photos.

\section{Availability of data and material}

The data used and/or analyzed in this case report are available from the corresponding author upon a reasonable request and following strict compliance with the regulations of personal data protection the author

\section{Author's Contribution}

\section{CR-P}

This author conceived the anesthetics technique and collected the data and consent informed document. Made the X-ray/Echo correlation of Spine features and helped to write the manuscript. Coordination and revising it for important intellectual content. CR-P approved the final manuscript.

\section{ZHS}

This author helped to do the anaesthetic technique and helped to write the manuscript. Coordination and revising it for important intellectual contect. ZHS approved the final manuscript.

\section{Acknowledgement}

We would like to thanks Dr. José Moreno Isturiz, Chief-Director of Labor and Delivery Room of Clínica El Avila and Dr. Salvador Malavé Quintero, Chief of Radiology Department of Clínica El Avila for all the support he gave us and patience.

\section{References}

1. Hawkins JL, Koonin LM, Palmer SK, Gibbs CP (1997) Anesthesia-related deaths during obstetric delivery in the United States, 1979-1990. Anesthesio 86: $277-284$.

2. Soleiman J, Demaerel P, Rocher S, Maes F, Marchal G (2005) Magnetic resonance imaging study of the level of termination of the conus medullaris and the thecal sac: Influence of age and gender. Spine 30: 1875-1880.

3. Arzola C, Davis S, Rofaeel A, Carvalho J (2007) Ultrasound using the transverse approach to the lumbar spine provides reliable landmarks for labor epidural. Anesth Analg 104: 1188-1192.

4. Carvalho JCA (2008) Ultrasound-facilitated epidurals and spinals in obstetrics Anesthesiol Clin 26: 145-158.

5. Grau T, Leipold RW, Horter R, Conradi R, Martin E, et al. (2001) Ultrasound imaging facilitate localization of the epidural space during combined spinal and epidural anesthesia. Reg Anesth and Pain Med 26: 64-67.

6. Lirk P, Moriggl B, Colvin J, Keller C, Kirchmair L, et al. (2004) The incidence of lumbar ligamentum flavum midline gaps. Anesth Analg 98: 1178-1180.

7. Lee Y, Tanaka M, Carvalho JCA (2008) Sonoanatomy of the lumbar spine in patients with previous unintentional dural punctures during labor epidurals. Reg Anesth Pain Med 33: 266-270.

8. Fettes P, Jansson J, Wildsmith J (2009) Failed spinal anaesthesia: Mechanisms, management, and prevention. Br J Anaesth 102: 739-748.

9. Chin KJ, Macfarlane A, Chan V, Brull R (2009) The use of ultrasound to facilitate spinal anesthesia in a patient with previous lumbar laminectomy and fusion: A case report. J Clin Ultrasound 37: 482-485.

10. Majeed A, Ahmed I, Alkahtani GJ, Altahtam NA (2017) Ultrasound-guided continuous spinal anesthesia for cesarean section in a parturient with scoliosis corrected with Harrington's rod surgery. Saudi J Anaesth 11: 479-482.

11. Tran D, Kamani AA, Al-Attas E, Lessoway VA, Massey S, et al. (2010) Singleoperator real-time ultrasound-guidance to aim and insert a lumbar epidural needle. Can J Anesth 57: 313-321. 